\title{
e-Migrinter
}

16 | 2017

Récits d'exilés

\section{Calais, Automne 2015 - Hiver 2016}

\section{Florence Prudhomme}

\section{(2) OpenEdition}

Journals

Édition électronique

URL : https://journals.openedition.org/e-migrinter/968

DOI : 10.4000/e-migrinter.968

ISSN : 1961-9685

\section{Éditeur}

UMR 7301 - Migrinter

\section{Référence électronique}

Florence Prudhomme, «Calais, Automne 2015 - Hiver 2016 », e-Migrinter [En ligne], 16 | 2017, mis en ligne le , consulté le 20 mai 2021. URL : http://journals.openedition.org/e-migrinter/968 ; DOI : https:// doi.org/10.4000/e-migrinter.968

Ce document a été généré automatiquement le 20 mai 2021

Tous droits réservés 


\section{Calais, Automne 2015 - Hiver 2016}

\section{Florence Prudhomme}

1 Septembre 2015, grande manifestation à Calais. Au départ de la «jungle». Il y a beaucoup de monde aux côtés des Soudanais, des Afghans, des Syriens, des Erythréens, des Kurdes... Sur leurs pancartes : «I am... », suivi de leur nom, de leur prénom, de leur pays. Et aussi «I am a friend », «I am Doctor », «I am a free man ». Des slogans : « We are Humans», "Houria ${ }^{1}$ ». Musique et danse jusqu'au port. Une manifestation de femmes également : «We are not animals », «Where are our rights? » Il y a beaucoup de soleil ce jour-là. L'hiver n'a pas encore commencé. La « jungle » se développe depuis plusieurs mois sur un terrain attribué aux réfugiés par l'Etat, dans une zone classée Seveso $^{2}$. L’Ecole laïque du Chemin des dunes a été inaugurée le 11 juillet.

Figure $\mathrm{n}^{\circ} 1$ : Manifestation de solidarité avec les réfugiés, Calais.

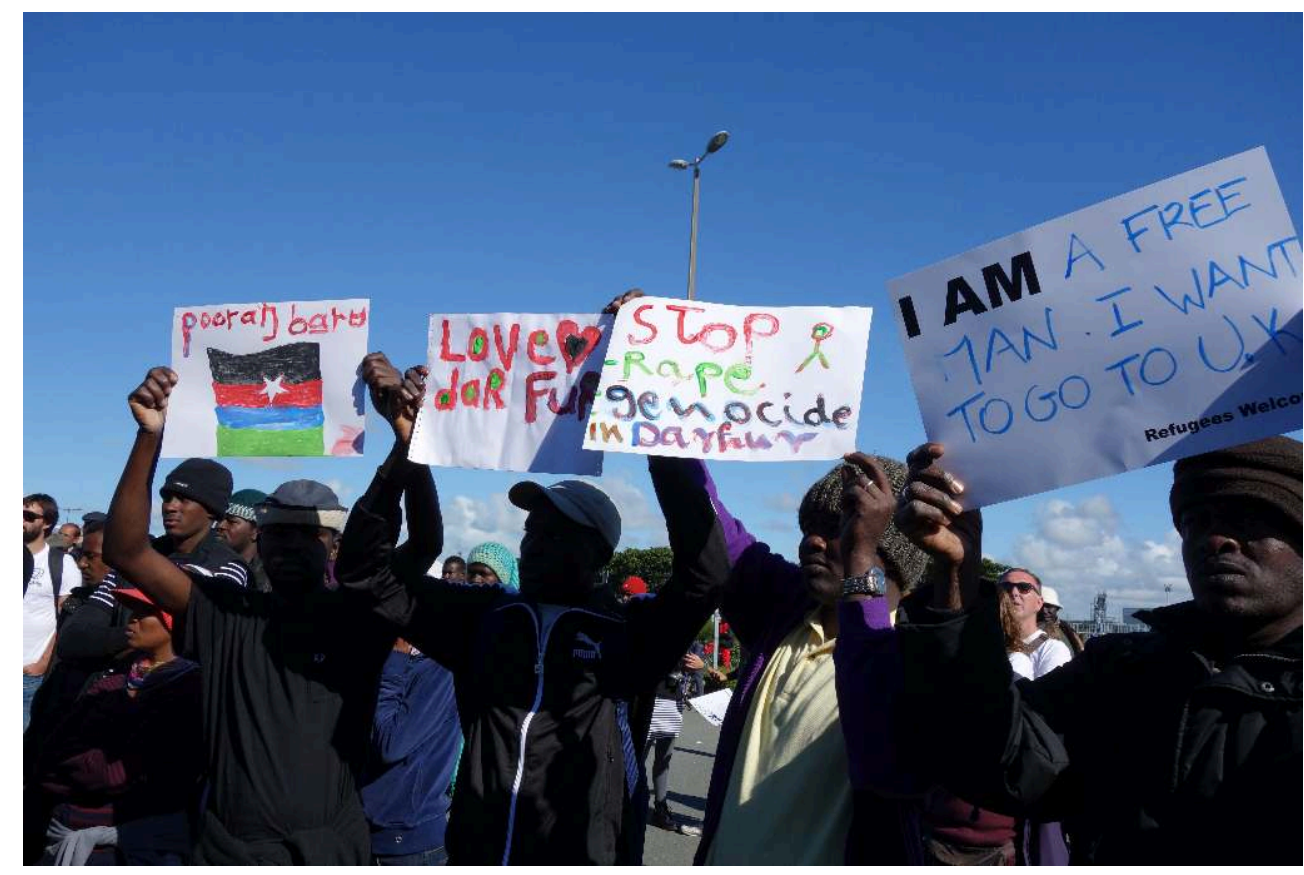

Florence Prudhomme, 19 septembre 2015. 
2 Au cours des mois suivants, les journées deviennent pluvieuses. Un vent violent transperce les toiles des tentes. La boue envahit une grande partie du campement. Des magasins, des églises, des mosquées sont construites. Une petite ville est née. Insalubre certes, mais inventive et solidaire. Dix mille personnes y vivent. Des initiatives surgissent comme autant de liens : expositions d'œuvres d'art, théâtre, salles de classes, bibliothèques, cinéma...

En décembre, avec des ami-e-s et des bénévoles responsables de l'Ecole laïque du Chemin des dunes, j'ai créé la Bibliothèque vivante. Les relations sont emplies de confiance, simples, chaleureuses. Mais le paysage est mouvant, instable. Pour les réfugiés, les nuits se passent à tenter de rejoindre l'Angleterre. Les matins sont calmes, ceux qui ont tenté en vain le passage s'efforcent de dormir jusqu'à la prochaine et périlleuse tentative.

4 Fin février 2016, c'est le coup d'envoi du démantèlement de la zone sud de la « jungle ». Des Iraniens se cousent les lèvres, une centaine de mineurs « disparaissent ». Toute la mise en scène préfigure le démantèlement définitif qui aura lieu huit mois plus tard.

Figure $n^{\circ} 2$ : Carte de la « jungle » de Calais, indiquant la zone sud.

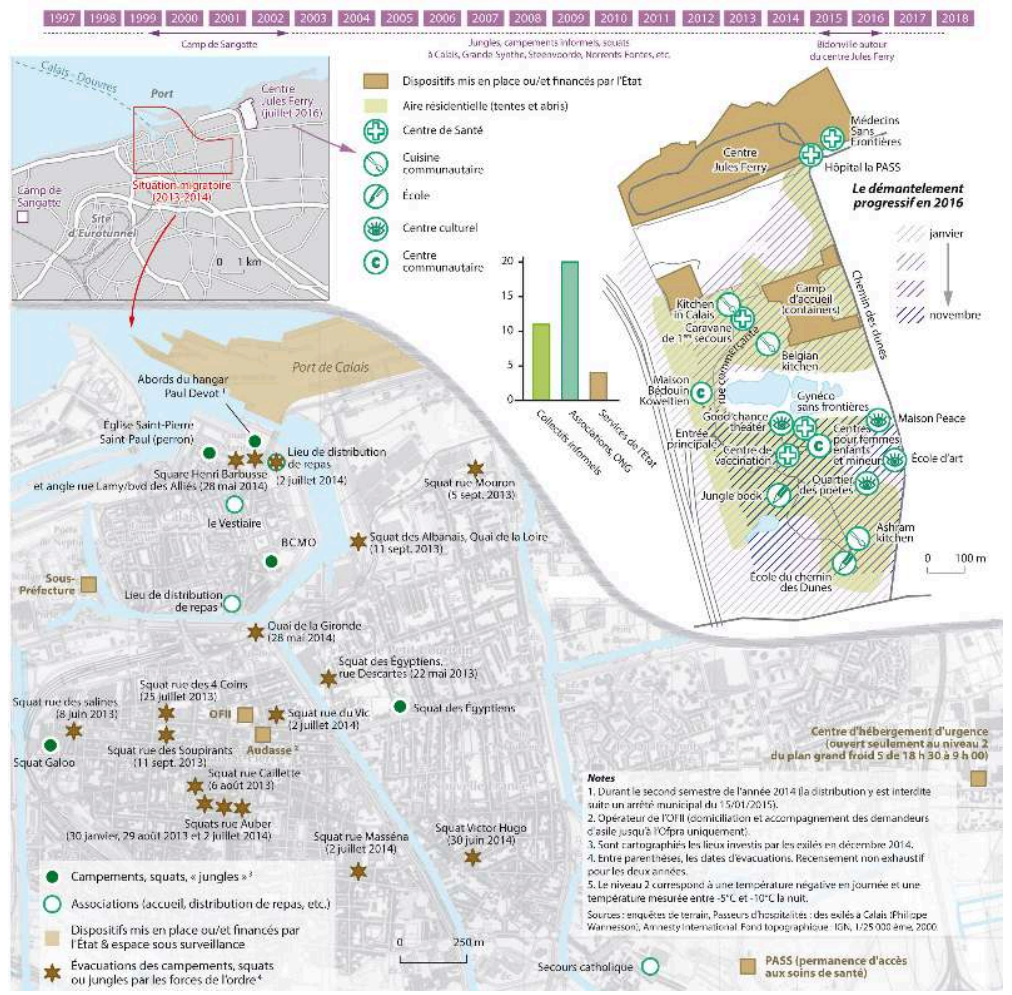

Sources : enquêtes de terrain ; Passeurs d'hospitalités : des exilés à Calais (Philippe Wannesson) ; Bertille Bouclier (2016) Que conditionne le développement de phénomènes auto-organisationnels des migrants dans le cas de Calais, et quelles peuvent être leurs retombées sur l'expérience de transit par la «jungle »? Mémoire de Master 1 « Migrations internationales » (Migrinter /Université de Poitiers) sous la direction de Céline Bergeon, 103 p. ; « Cartographie 2017 des engagements volontaires et solidaires à l'international », Ivry-sur-Seine, France Volontaires ; Document de l'association « L'Auberge des migrants » sur la présence associative à Calais durant le premier semestre 2016 ; Amnesty International.

Cette carte a été conçue par Olivier Clochard et Olivier Pissoat. Migreurop (2017) Atlas des migrants en Europe: Approches critiques des politiques migratoires, Paris, Armand Colin, p. 153. 

mineurs privés des droits qui sont les leurs, tri au faciès qui les concerne en premier lieu, dévastation. Les réfugiés sont emmenés dans des cars, dispersés dans tout le pays par petits groupes. Où sont-ils? Disparus de nos yeux. L'objectif est atteint : ils ne sont plus visibles, ils n'existent plus. A Creil dans l'Oise, Fouad, réfugié syrien profondément traumatisé, dit : "Toutes les routes se ferment devant nous ». Il a vécu un an dans la «jungle» de Calais, avant de se retrouver à Paris d'où il a été chassé lors d'un démantèlement des campements du quartier de Stalingrad pour finir dans un sinistre Centre d'accueil et d'orientation (CAO) ${ }^{3}$. Une attestation, délivrée par un praticien de Médecins sans frontières, confirme qu'il est nécessaire de poursuivre sa prise en charge psychologique. Mais là, à Creil, il n'a pas d'ami, il n'y a pas de bénévoles : « Ici, je n'ai personne", "c'est impossible d'avoir quelqu'un qui t'accompagne chez le docteur » (Extrait du récit de Fouad, novembre 2016).

Quelques municipalités (par exemple Croisilles dans le Pas-de-Calais ou Allex dans la Drôme) et des universités (Lille, Grenoble) accueillent chaleureusement et dignement les réfugiés. Un ami afghan est accueilli par une bergère dans les Alpes de Haute Provence. Le lendemain de son arrivée, il part faire la transhumance et s'occupe des ânes qu'il connaît bien.

Dans d'autres villes, notamment à Calais et à Paris, après le démantèlement du campement de Stalingrad et de l'avenue de Flandre, la chasse au faciès se renforce, les menaces de reconduites dans des pays en guerre (Afghanistan, Irak, Soudan) se profilent avec insistance. Des personnes qui ont aidé des réfugiés sont convoquées devant les tribunaux. Le délit de solidarité s'installe.

Dans ce climat policier et violent, nous avons recueilli dans la « jungle » une quinzaine de récits dans les langues d'origine des réfugiés (pashto, dari, arabe). Les trois textes qui suivent comprennent deux textes de Hameedullah Yadgary, Nothing et La vie Jungle, confiés à la Bibliothèque vivante et travaillés avec l'auteur. Né à Jalalabad en Afghanistan, âgé aujourd'hui de 30 ans, Hameedullah a obtenu le droit d'asile en juillet 2016. Le troisième texte, écrit par Omar, jeune Soudanais, âgé de 21 ans, est issu d'un entretien en arabe avec la Bibliothèque vivante. Le démantèlement total du campement de Calais en octobre 2016 et la dispersion dans la France entière de ceux qui vivaient là ont considérablement réduit la possibilité de poursuivre les entretiens. Ces textes sont destinés à rendre visibles et audibles ceux qui sont réduits à l'appartenance à un groupe indistinct de personnes - « eux », privées d'identité et de subjectivité.

\section{BIBLIOGRAPHIE}

Le récit de Fouad, perdu à Creil, Jungle News, 5 novembre 2016 [Disponible sur Internet]

La « jungle » de Calais est majoritairement située en zone Seveso, Le Monde, réf. du 19 octobre 2015 [Disponible sur Internet]

e-Migrinter, 16 | 2017 
Prudhomme, Florence (2016) " Je naviguais vers mon espoir ", in Décamper, De Lampedusa à Calais, un livre de textes et d'images \& un disque pour parler d'une terre sans accueil, Paris, La Découverte, pp. 92-97.

Prudhomme, Florence (2015) Rien de l'autre ne m'est étranger, Mediapart, réf. du 29 décembre 2015 [Disponible sur Internet]

Prudhomme, Florence (2016) La Bibliothèque vivante de la « jungle » de Calais, Mediapart, réf. du 28 janvier 2016 [Disponible sur Internet]

Prudhomme, Florence (2016) Une petite ville est née..., Mediapart, réf. du15 février 2016

[Disponible sur Internet]

Prudhomme, Florence (2016) Apprendre de Calais : entretien avec Cyrille Hanappe, Mediapart, réf. $\mathrm{du} 9$ juillet 2016 [Disponible sur Internet]

Prudhomme, Florence (2016) Non-assistance à personnes et mineurs en danger, Mediapart, réf. du 28 octobre 2016 [Disponible sur Internet]

\section{ANNEXES}

\section{La vie « Jungle »}

Hameedullah Yadgary, originaire d'Afghanistan, 29 ans. Traduit de l'anglais par Florence Prudhomme, 2016.

C'est le matin. Vous devez vous réveiller, vos vêtements sont trempés par la rosée parce que vous dormez dans une tente qui ne vous protège ni du froid ni du vent ni de la pluie. Vous devez vous réveiller de bonne heure et vous rapprocher de la porte de l'association Salam ${ }^{4}$ et attendre jusqu'à ce que la porte s'ouvre. Tout le monde veut passer devant et pousse les autres pour être au plus près de la porte. Quelqu'un arrive et ouvre la porte. On dirait une course de chevaux, tout le monde accourt et se bouscule violemment pour être parmi les premiers et ne pas rester pendant des heures dans le froid ou sous la pluie battante. Certains tombent. Tout le monde les ignore, continue son chemin et avance. Ceux qui sont derrière doivent rester dans la file pendant deux ou trois heures s'ils veulent prendre une douche. Qu'il fasse un temps glacial ou qu'il pleuve, vous devez continuer à faire la queue. Juste pour prendre une douche qui est réglée pour durer six minutes, et pas une de plus.

\section{Un repas en vingt-quatre heures}

En vingt-quatre heures, vous recevez un seul repas et pour le recevoir vous devez attendre deux à trois heures dans la file. Parfois vous le ratez parce que vous êtes trop fatigué pour tenir debout et vous attendez vingt-quatre heures pour le prochain repas, en pensant à ce que vous aurez. Si vous avez de l'argent, pas de problème. Vous pouvez manger dans les restaurants de la jungle ou cuisiner si vous avez la chance de trouver quelque chose à cuire. Mais beaucoup attendent le ventre vide durant tout le reste de la journée et de la nuit. Au début j'avais un peu d'argent mais après quelques jours, j'avais du mal à trouver ne serait-ce qu'un euro pour m'acheter à manger. Un de mes amis travaillait dans un restaurant, j'allais là-bas et j'attendais que les gens aient fini de 
manger. S'ils laissaient quelque chose dans leur assiette, je mangeais les restes après leur départ. Parfois mon ami me donnait quelque chose à manger. Régulièrement j'allais dans le restaurant pour recharger mon portable et parler avec ma famille, pour qu'elle me redonne confiance. Sinon j'aurais perdu tout espoir. Un jour je parlais avec ma mère, au bout d'une minute je n'ai pas pu me contrôler et je me suis mis à pleurer. Je suis sorti sous la pluie pour que personne ne remarque que j'étais en larmes. Tout le monde a vécu des histoires horribles. La nuit, chacun perdait espoir et ses rêves s'écroulaient. Mais il n'y avait pas d'autre choix.

\section{Nuits effrayantes}

Quand le ciel devient sombre, les réfugiés tentent de nouveau leur chance, ils marchent pendant trois à quatre heures pour rejoindre le parking où stationnent les camions qui sont en partance vers l'Angleterre. Chacun essaie de se glisser à l'intérieur, mais cela prend des heures pour y parvenir et il faut surmonter toutes sortes d'obstacles. Est-ce que vous imaginez ce que c'est que de se trouver sous un camion, près des roues pendant cinq à dix heures? C'est un risque énorme. Nous ne l'avons pas fait une fois, nous ne l'avons pas fait deux fois, nous l'avons fait un nombre incalculable de fois. Vraiment c'est effrayant et trop dur de se tenir ainsi. Après avoir réussi à entrer à l'intérieur du camion ou à se glisser en-dessous, il faut attendre des heures dans le froid et dans des conditions terribles. Beaucoup essaient tous les soirs. Certains ont mangé, d'autres ont faim, d'autres sont malades, d'autres courent juste pour avoir chaud, et ils essaient encore et encore. Soudain le conducteur ou quelque citoyen " respectable » nous voit et appelle la police. Vous êtes faits. Vous avez passé toute la nuit dans le froid pour rien. La police court derrière vous, elle vous asperge de gaz lacrymogène, elle vous blesse, elle vous frappe avec des câbles électriques et cherche à vous nuire de toutes les manières possibles. Parfois on se dit que peut-être nous ne sommes pas des êtres humains, parce que personne ne traite un humain comme ça.

Après vingt à trente minutes de poursuite sous une pluie de gaz lacrymogène, le réfugié assoiffé, épuisé, a perdu tout espoir. Il reprend le chemin en sens inverse et repart vers la « jungle » avec des larmes dans les yeux et le visage empreint de fatigue. Il marche pendant des heures jusqu'à la jungle. D'autres passent toute la nuit à l'intérieur du camion. Le matin le conducteur arrive et il dit : «Sortez, je sais que vous êtes là. » Certains s'en rendent compte lorsqu'ils vérifient le camion avant de partir. Un nombre infime de réfugiés aura la chance de connaître la fin de ses souffrances.

De retour dans votre tente vous ne pouvez pas même vous mettre sous la couverture tant elle est froide et humide. Et vous vous souvenez de votre pays, de votre maison, de votre lit. Vos yeux se remplissent à nouveau de larmes, mais vous devez dormir ou trouver quelque chose à manger. Vous ne pouvez rien préparer tellement vous êtes fatigué mais vous devez quand même vous en préoccuper. Vous avez aussi besoin de dormir parce qu'à n'importe quel moment il sera possible de recommencer et d'essayer encore. Et passer encore toute la nuit dehors. Nous nous forçons à dormir, mais dans nos rêves on continue encore à tenter de passer. Souvent je rêve que je réussis à rejoindre l'Angleterre... Mais on ne peut pas dormir trop longtemps, on doit se lever pour se mettre en ligne et faire la queue pour recevoir un repas, des vêtements, des chaussures ou rapporter quelque chose à manger et être prêt pour la prochaine horrible nuit. Chaque seconde je pense à ce qui pourrait m'arriver, je pense à mon 
destin. Est-ce que je vais survivre ou bien mourir, un jour ou une nuit, comme quelques-uns de mes amis? Qu'est-ce qui se passera si je meurs et que personne ne me reconnaît? Et que se passera-t-il pour ma mère qui prie sans cesse pour moi ? Elle qui reste en vie pour me revoir encore une fois, elle ne me verra plus jamais et son attente ne prendra jamais fin. Quelquefois j'ai l'impression d'avoir une grosse pierre sur la poitrine, soudain je me mets à pleurer et j'appelle ma mère et j'appelle Dieu pour qu'il me vienne en aide.

Pour Dieu, nous aussi nous sommes des humains, exactement comme tout un chacun dans ce monde. Nous ne sommes pas ici par choix. Ce sont les conditions qui règnent dans nos pays qui nous ont mis dans cette situation. Nous ne voulons pas de grandes maisons, des voitures de luxe ou plus d'argent, nous voulons juste une vie simple dans la paix et la dignité. L'atmosphère dans la jungle est affectueuse comme celle d'une famille. Chacun aime l'autre même s'il y a de grandes différences entre nos cultures et nos traditions. Chaque pays a sa propre localisation, mais parfois on devient ennemis les uns des autres jusqu'à vouloir s'entretuer. Ce sont les frustrations et les cauchemars éprouvés jour après jour et nuit après nuit qui en sont la cause.

\section{Alors maintenant}

Après avoir passé quatre horribles mois dans la « jungle ",j'ai renoncé et j'ai perdu tout espoir de rejoindre l'Angleterre.

Un jour je suis allé là où se trouvent les bus qui emmènent les réfugiés dans les Centres d'accueil et d'orientation (CAO) ${ }^{5}$. Ils m'ont conduit à Istres dans le sud de la France. Nous sommes arrivés là-bas le matin après vingt heures de trajet. Nous avons vu les autorités qui nous attendaient. L'accueil a été chaleureux et ils nous ont donné un peu de nourriture. Après un check-up médical, on nous a accompagnés dans nos chambres. Une chambre propre et chauffée comme je n'en avais pas vu pendant les quatre derniers mois. J'ai pris une douche et je suis allé prendre mon repas avec les autres. Cette nuit-là j'ai dormi en paix, dans un lieu où il n'y avait ni tensions ni vents mauvais, ni pluie ni froid, ni gaz lacrymogène ni violences. Maintenant il y a dix mois que je suis dans ce centre. J'ai obtenu le statut de réfugié. J'apprends le français et je suis heureux. Si j'avais su ça plus tôt, je n'aurais jamais passé tout ce temps dans la « jungle ». Maintenant chaque fois que je me souviens de la « jungle » et de ces mauvais jours, les larmes me viennent aux yeux. J'ai beaucoup de mauvais souvenirs et quelques rares bons souvenirs de la « jungle », je ne les oublierai jamais. J'espère que plus personne n'aura à vivre des jours pareils, si durs et si terribles. J'espère un monde sans frontières ni barrières qui séparent les humains les uns des autres.

\section{Nothing}

Hameedullah Yadgary, originaire d'Afghanistan, 29 ans. Traduit de l'anglais par Florence Prudhomme, 2016

Vous avez 29 ans, une épouse, deux enfants et un métier. Vous avez suffisamment d'argent pour vivre et vous offrir quelques jolies choses, et vous vivez dans une petite maison dans la ville. 
Mais soudain la situation politique dans votre pays change et quelques mois plus tard des soldats stationnent devant votre maison. Et devant les maisons de vos voisins.

Ils disent que si vous ne vous battez pas pour eux, ils vont vous tirer dessus. Votre voisin refuse. Un tir. C'est tout.

Vous surprenez un des soldats qui dit à votre femme d'allonger ses jambes.

Sans savoir comment, vous échappez aux soldats et vous passez la nuit profondément perdu dans vos pensées. Soudain, vous entendez une explosion. Votre maison n'a dorénavant plus de living room. Vous courez à l'extérieur et voyez que toute la rue est détruite. Plus rien ne tient debout.

Vous faites rentrer votre famille dans la maison, et vous courez jusqu'à la maison de vos parents.

Elle n'existe plus. Et vos parents pas davantage. Vous regardez autour de vous et trouvez un bras avec l'anneau de votre mère à son doigt. Vous ne trouvez aucun autre signe de vos parents.

«Mais les demandeurs d'asile ont tant de produits de luxe! Des smartphones et des vêtements de marque!»

Vous oubliez immédiatement. Vous vous précipitez à la maison, et dites à votre femme d'habiller les enfants. Vous prenez un petit sac, parce qu'il est impossible d'en prendre un plus grand pour un si long voyage. Vous emportez l'essentiel. Le sac ne contient pas plus de deux vêtements pour chacun. Que prendre?

Vous ne reverrez probablement jamais votre pays d'origine. Ni votre famille, ni vos voisins, ni vos collègues... Mais comment rester en contact? Vous jetez vite votre smartphone et le chargeur dans le sac. Avec les quelques vêtements, un peu de pain et les peluches préférées de vos petites filles.

«Ils peuvent facilement se permettre de partir. Ils ne sont pas pauvres !»

Parce que vous avez vu le danger s'approcher, vous avez rassemblé tout l'argent que vous avez réussi à économiser grâce à votre emploi bien rémunéré. Les passeurs dans le quartier prennent 5000 euros par personne. Vous avez 15000 euros. Avec un peu de chance, vous serez tous en mesure de partir. Sinon, vous laisserez votre femme partir. Vous l'aimez et priez pour que les passeurs vous emmènent tous.

À cet instant-là, vous êtes totalement démuni et vous n'avez rien d'autre. Juste votre famille et le sac.

Le voyage jusqu'à la frontière dure deux semaines à pied. Vous avez faim ; au cours de la semaine passée vous avez très peu mangé. Vous êtes faible, votre femme aussi. Mais vos deux petites filles ont pu manger suffisamment.

Elles n'ont cessé de pleurer tout au long de ces deux semaines. La moitié du temps vous avez porté votre fille cadette. Elle est âgée seulement de 21 mois.

Deux semaines plus tard vous arrivez au bord de la mer. Au milieu de la nuit, vous êtes chargés sur un bateau avec d'autres réfugiés. Vous avez de la chance : toute votre famille peut voyager. L'embarcation est tellement pleine qu'elle menace de chavirer. Vous priez et suppliez de ne pas être noyés. 
Les gens autour de vous pleurent et crient. Quelques petits enfants meurent à cause de la soif. Les passeurs les jettent par-dessus bord. Votre femme est assise dans un coin, hébétée. Elle n'a rien eu à boire depuis deux jours. Lorsque la côte est en vue, vous êtes chargés sur des bateaux plus petits. Votre femme et l'enfant la plus jeune sont sur l'un, vous et l'enfant plus âgée sur un autre.

On vous avertit que vous devez garder le silence afin que personne ne sache que vous êtes là. Votre fille aînée comprend. Mais la plus jeune dans l'autre bateau ne comprend pas. Elle ne cesse de pleurer. Les réfugiés deviennent nerveux. Ils demandent à votre femme de calmer l'enfant. Elle n'y parvient pas. Un des hommes attrape votre fille, l'arrache des bras de votre femme et la jette par-dessus bord.

Vous sautez derrière elle, mais vous ne pouvez pas la retrouver. Plus jamais. Trois mois plus tard, elle aurait eu 2 ans.

«Est-ce que ce n'est pas suffisant pour vous ? Est-ce que ce n'est pas assez bien, est-ce que tout ne vous est pas servi sur un plateau?»

Vous ne savez pas comment vous, votre femme et votre fille aînée parvenez à entrer dans ce pays où vous arrivez. Tout semble recouvert de brume. Votre femme n'a pas dit un mot depuis que votre fille est morte. Votre fille aînée n'a pas lâché la peluche de sa sœur et elle est totalement apathique.

Mais il faut continuer. Vous êtes sur le point d'arriver à l'hébergement d'urgence. Il est 10 heures du soir. Un homme dont vous ne comprenez pas la langue vous emmène dans une salle avec des lits de camp. Il y a cinq cent lits très proches les uns des autres. Dans la salle, c'est étouffant et bruyant. Vous essayez de prendre vos repères, de comprendre ce que les gens ici attendent de vous. Mais en réalité, vous pouvez à peine vous tenir debout. Vous préféreriez qu'ils vous aient tué. Au lieu de ça vous déballez vos maigres possessions : les deux sortes de vêtements pour chacun et votre smartphone.

Vous passez votre première nuit dans un pays sûr. Le lendemain matin, on vous donne quelques vêtements. Parmi lesquels des vêtements « de marque ». Et un jouet pour votre fille. On vous donne 140 euros. Pour tout le mois.

« Ils sont en sécurité ici. Ils devraient être heureux!»

Dehors, dans la cour, vêtu de nouveaux vêtements, vous tenez votre smartphone en l'air en espérant avoir la connexion. Vous voulez savoir si quelqu'un dans votre ville est encore en vie. Puis arrive un citoyen « respectable», il vient vers vous et vous injurie. Vous ne savez pas pourquoi. Vous ne comprenez pas.

« Retournez dans votre pays!»

Vous comprenez quelque chose comme « smartphone » et « tout servi sur un plateau ». Quelqu'un traduit pour vous.

Et maintenant, dites-moi comment vous sentez-vous ? Que possédez-vous ? La réponse aux deux questions est « Rien » (Nothing).

\section{« Je n'ai jamais vu le bonheur... »}

Omar, originaire du Darfour (Soudan), 21 ans, texte recueilli par la Bibliothèque vivante. Traduit de l'arabe par Kamel Hamdi. 
J'habitais dans un petit village au Darfour. On ne connaissait pas la modernité. C'était la vie traditionnelle : on avait des moutons et des vaches, quelques ânes, et on semait le grain. Je n'ai pas beaucoup étudié, je suis allé à l'école coranique pendant trois ans, de 12 à 16 ans. Pendant toutes ces années je ne suis jamais sorti de mon village. La vie était stable avec des récoltes saisonnières de légumes et de tomates à l'automne. Quand il y avait une bonne récolte, on en conservait une partie en prévision de l'année suivante, si jamais la pluie venait à manquer. On buvait le lait des vaches, mais on ne le vendait pas. On faisait du beurre ou des fromages traditionnels en gardant le lait trois jours dans une peau de mouton. On mélangeait tout ça avec le couscous. De décembre à avril, pendant la saison où on ne travaille pas, il y avait des fêtes et des mariages.

En 2004, je vivais à Hilla avec ma famille. Mon père et ma mère restaient là pendant que mon frère et moi quittions la maison pour nous rendre plus loin, dans un autre village, à Farig, où on conduisait les moutons dans un grand champ avec des herbes. Le soir on rentrait pour faire boire le troupeau. Un jour les miliciens Janjawids ${ }^{6}$ sont arrivés, ils ont détruit tout le village, en commençant par la réserve d'eau. En rentrant ce soir-là, les bêtes n'ont pas pu boire, car la citerne était hors d'usage. On a perdu la moitié du troupeau.

Après cette attaque tout le monde s'est dispersé. Mon père âgé de 70 ans avait deux épouses. Avec la plus âgée, il est parti au Tchad. Puis il est revenu pour chercher ses deux fils et les moutons. En chemin il s'est fait agresser par la police du régime et il est allé en prison pendant une semaine. A Hilla la police interrogeait ceux qui étaient sur place : «Où sont les rebelles? » La police posait des questions que personne ne comprenait, elle obligeait les gens à dire des choses qu'ils ne comprenaient pas. « Les rebelles ", personne ne savait ce que ça voulait dire. Quand mon père est arrivé, une semaine plus tard, il n'a retrouvé aucun de nous. Ma mère - sa seconde épouse, mon petit frère et moi, étions partis plus loin. Nous avions pris des ânes pour sortir du village et nous avions voyagé pendant un jour et demi à dos d'âne. Nous n'avions rien d'autre à manger que du lait ou du lait battu, et des figues de Barbarie quand on avait la chance d'en trouver. Nous sommes restés dans ce village, à Orori. Nous avons passé deux jours sous les arbres sans rien.

Nous sommes restés là d'octobre à fin juillet. J'avais 14 ou 15 ans et mon petit frère en avait 12. La première épouse économisait ses denrées et nous faisait parvenir du savon et d'autres produits de première nécessité. Des associations nous aidaient un peu aussi.

Nous avons décidé de repartir à Hilla. Le village comptait autrefois une soixantaine d'ânes, un an plus tard il n'en restait que deux. Tout ce que nous avions avait été détruit, nous n'avions même plus d'extraits d'acte de naissance pour connaître notre âge. C'était l'automne, après la saison des pluies. Ma mère et mon petit frère ont cultivé chez des gens. Moi, je suis parti pour garder des ânes. Pour une année de travail, mon salaire était de deux ânes. De 2005 à 2007, je suis resté durant ces trois années à la même place, sans voir mon père, ni ma mère, ni mon petit frère. J'ai gagné six ânes. Ma mère a chanté, elle a dansé... Six ânes, c'était une fortune

En 2008 je suis parti avec mes ânes pour rejoindre ma famille à Hilla. Ma mère a chanté, elle a dansé, elle était contente. Six ânes, c'était une fortune. La situation est restée à peu près stable jusqu'en 2015. Mais le 17 janvier nous avons connu de nouveau une 
attaque des Janjawids. J'ai perdu mes six ânes. Nous avions tout perdu, il ne nous restait rien. Même la nourriture économisée par ma mère avait été pillée.

Nous avons de nouveau quitté Hilla. Nous sommes allés vers la frontière avec le Tchad, où se trouvait un camp de l'UNICEF. Mes parents sont peut-être toujours dans ce camp mais je n'ai aucune nouvelle d'eux. Je les ai perdus à ce moment-là. Je suis resté seul sur place pendant deux mois. Des camions venaient régulièrement de Libye pour chercher des ânes. Des personnes m'ont dit de venir avec les ânes. C'étaient des passeurs pour la Libye, comme il y en a ici pour rejoindre l'Angleterre.

Je me suis décidé à partir comme mes amis d'enfance l'avaient fait avant moi. Je suis parti pour avoir à boire et à manger, être en paix dans un pays sécurisé. La Libye, c'était une issue de secours. Je suis resté à Benghazi cinq mois. Mon ami, Zouara, m’a dit : « La Sicile, c'est tout près ». Nous avons payé environ 600 euros et avons pris une petite embarcation.

Très vite il y a eu plein d'eau dans le bateau. Le moteur s'est arrêté. Nous avons écopé l'eau. On attendait la mort. On regardait le ciel. Soudain nous avons vu un hélicoptère. Il nous a repérés et a demandé l'aide d'un bateau de secours en Sicile. Ils ont secouru les petits enfants, puis les femmes, puis nous. La mer était tellement mauvaise que le bateau de secours est resté deux jours supplémentaires en mer pour sauver d'autres petits bateaux. Ils en ont secouru deux.

Nous avons été bien accueillis en Sicile. On nous a donné à boire et des vêtements. Trois jours après on nous a mis dans des bus pour l'Italie. À Milan, j'étais avec un ami qui parlait anglais. Nous avons rejoint Vintimille. La Croix-Rouge nous a donné à manger et des vêtements. Nous avons vu le médecin. Par le train nous sommes allés à Nice, puis Grenoble, Lyon, Paris. À Paris, nous sommes allés vers la Porte de la Chapelle. Durant quatre jours nous sommes restés dans une école où il y avait d'autres réfugiés. Puis on a rejoint Calais il y a dix mois.

J'espère toujours passer en Angleterre. Mais j'ai tout le temps peur.

En septembre 2015, dans la " jungle », je dormais dans une petite tente avec mon ami. Près de la grande tente des Soudanais. À 2 heures du matin une voiture a roulé sur notre tente. Mon ami a été grièvement blessé, il a passé deux mois à l'hôpital de Calais. Au bout de deux jours j'ai demandé des informations aux voisins de notre tente. La voiture était toujours là. Le conducteur avait pris la fuite. Une avocate a étudié mon dossier, elle est allée à la police qui ne lui a donné aucune information. Mon ami a fait une demande d'asile, elle lui a été refusée.

\section{NOTES}

1. «Liberté » en arabe.

2. La TCDD, surnommée dioxine de Seveso, est l'une des molécules les plus toxiques pour l'homme et la seule considérée comme cancérigène. La catastrophe de Seveso s'est produite le 10 juillet 1976. Un nuage contenant de la dioxine s'est échappé d'un réacteur de l'usine chimique Icmesa, située dans la commune de Meda. Il s'est répandu sur la plaine lombarde en Italie. Quatre communes, dont Seveso, ont été touchées. Jusqu'au 23 juillet, la population a continué à vivre dans un milieu contaminé. Le 26 juillet, deux cent vingt-cinq habitants sont évacués, suivis de cinq cent autres quelques jours plus tard. Suite à cette catastrophe et à sa gestion bâclée, les États 
européens ont réalisé qu'il était nécessaire de renforcer le contrôle des pouvoirs publics sur les activités industrielles présentant des risques technologiques majeurs. La catastrophe a donné naissance en Europe à la Directive Seveso en 1982. Puis à la Directive Seveso 2 en 2000 et à la Directive Seveso 3 en 2012.

3. Les Centres d'aide et d'orientation (CAO) ont été institués par le ministre de l'Intérieur, Bernard Cazeneuve, en novembre 2015. Ils sont censés leur permettre de réfléchir à leur vie future et éventuellement d'entreprendre des démarches de demande d'asile en France, si c'est leur souhait.

4. L'association Salam a été créée à la suite de la fermeture en novembre 2002 du centre de la Croix Rouge à Sangatte qui accueillait les réfugiés. Sans relâche elle leur assure depuis des distributions de nourriture et de vêtements.

5. Les Centres d'aide et d'orientation (CAO) ont été institués par le ministre de l'Intérieur, Bernard Cazeneuve, en novembre 2015. Ils sont censés leur permettre de réfléchir à leur vie future et éventuellement d'entreprendre des démarches de demande d'asile en France, si c'est leur souhait.

6. Les Janjawids sont des milices armées qui commettent des massacres, viols, enlèvements et pillages depuis 2003 au Darfour.

INDEX

Index géographique : France, Calais

Mots-clés : réfugiés, accueil, conditions de vie, récit, Soudanais, Afghans

\section{AUTEUR}

\section{FLORENCE PRUDHOMME}

Auteure de Rwanda, l'art de se reconstruire (Ateliers Henry Dougier, 2015), initiatrice de la Bibliothèque vivante à Calais

florence.prudhomme@wanadoo.fr 